\title{
Perspectives on Allyship in Academia
}

\author{
Jamie Payton \\ Temple University \\ Philadelphia, PA, USA \\ payton@temple.edu \\ Melissa Hovik, Kevin Lin \\ University of Washington \\ Seattle, WA, USA \\ \{medskm, kevinl\}@cs.uw.edu
}

\author{
Adam Blank \\ California Institute of Technology \\ Pasadena, CA, USA \\ blank@caltech.edu
}

\author{
Christian Murphy \\ University of Pennsylvania \\ Philadelphia, PA, USA \\ cdmurphy@seas.upenn.edu
}

\begin{abstract}
Allyship in academia is critical for creating inclusive communities that are welcoming to all students, but the perception of its benefits and challenges can vary depending on a number of factors. This session will explore perspectives of allyship in academia by bringing together a diverse group of faculty and students who can share a wide range of experiences and insights, and aims to facilitate a discussion among all attendees that leads to an exchange of ideas, the strengthening of our community, and progress toward our common goal of inclusion in computing.
\end{abstract}

\section{CCS CONCEPTS: • Social and professional topics $\sim$ User} characteristics • Social and professional topics Informal education - Social and professional topics Codes of ethics

KEYWORDS: Diversity; Inclusion; Belonging; Equity; Allyship

\section{OBJECTIVE}

Addressing the lack of representation and marginalization of various minority groups in computing is not solely in the purview of members of those groups: those who hold power and influence in our field have a responsibility to actively participate in solving these problems. As such, allyship movements in tech companies and organizations have begun to grow in number and in popularity, with allies advocating for members of underrepresented minority groups and for the creation of inclusive environments.

Allyship is necessary not only in industry, but in academia as well. Given the disproportionately low rate of retention among members of underrepresented groups in Computer Science majors, allyship -from faculty members and from student peers alike -- is crucial for providing individual mentorship and support, as well as for fostering inclusive communities that celebrate their contributions and provide a sense of belonging.

While there is little debate that allyship done well is a positive thing, the perception of its benefits and challenges will vary between faculty and students, and between men and women. Why engage in Permission to make digital or hard copies of part or all of this work for personal or classroom use is granted without fee provided that copies are not made or distributed for profit or commercial advantage and that copies bear this notice and the full citation on the first page. Copyrights for third-party components of this work must be honored. For all other uses, contact the Owner/Author.

SIGCSE '20, March 11-14, 2020, Portland, OR, USA

(๑) 2020 Copyright is held by the owner/author(s).

ACM ISBN 978-1-4503-6793-6/20/03.

https://doi.org/10.1145/3328778.3366997

\author{
Angela Kwon, Lara McConnaughey \\ University of California, Berkeley \\ Berkeley, CA, USA \\ \{kwonangela7, laram\}@berkeley.edu
}

allyship? What constitutes good allyship? How do we grow allyship in our departments? The answers to these questions will depend on whether one is considered an ally or has an ally (or both), and how they see themselves within the field of computing

Unlike allies panels that typically provide a single perspective -namely that of the ally -- this session seeks to explore the diverse perspectives of allyship in academia by bringing together faculty members and students who can share a wide range of experiences and insights. In addition to providing a broader perspective on academic allyship, this session will include time for the moderator to pose questions to the audience so that they too can contribute to the discussion and share their thoughts as well.

\section{OUTLINE}

Our goal is not to have a panel of so-called "expert allies" who seek to inform the audience about how to be an ally or to ask underrepresented groups to train allies, but rather to facilitate a discussion among all attendees that leads to an exchange of ideas, the strengthening of our community, and progress toward our common goal of inclusion in computing.

As such, this session will consist of three segments and will combine the benefits of the panel format and the Birds of a Feather format:

1. Opening Remarks (5 minutes): The moderator (Payton) will briefly introduce herself and the other organizers, and describe the goals of the session.

2. Moderator Questions for Organizers (25 minutes): The moderator will ask the other organizers questions about their experiences with allyship in academia.

3. Moderated Audience Discussion (45 minutes): The moderator will pose questions for the audience to answer about their experiences with allyship in academia. Organizers can also contribute answers to these questions as appropriate.During this time, questions from the audience can also be directed to individuals or the organizers in general.

The organizers each bring a different perspective to the notion of academic allyship:

- As the Director of the STARS Computing Corps Alliance for Broadening Participation in Computing, Payton brings a perspective on national resources and organizations for developing student and faculty allyship for students from 
underrepresented groups and leadership for broaden participation in computing. As a department chair, she also brings a perspective on how to support department-wide initiatives that foster positive growth in student and faculty allyship.

- Blank, Murphy, and Payton are faculty members who can speak to the efforts that faculty members can make toward allyship, including course- and research-related efforts, student mentorship, and departmental culture.

- Hovik is a current CS Master's student and predoctoral lecturer who can talk about the impact allies have and continue to have in her academic pathways and her current professional goals.

- Lin is a Lecturer who can address ways in which students can act as allies for other students, whether as classmates, teaching assistants, mentors, or advocates. He has conducted over 200 hours of classroom observations to understand how a defensive communication climate can form in undergraduate CS classrooms.

- Kwon is a recent UC Berkeley graduate who was involved in CS education in teaching, mentoring and research. She can talk about how impactful allyship (or the lack thereof) has been for herself and how she has tried to incorporate allyship in undergraduate courses.

- McConnaughey is a recent UC Berkeley graduate whose thesis work analyzed how undergraduate students' experiences with stereotypes and stigmatization in their first CS course influence whether or not they decide to continue learning CS.

Following are sample questions that the moderator may pose to the other organizers in Segment \#2:

- (To all): There are many cases in which well-intentioned efforts around allyship and advocacy in academia have failed. In what ways have you seen academic allyship fail? How can allies avoid and recover from such failures?

- (To all): Despite the growth of allyship in many CS departments, there are still vast numbers of faculty and students who resist being involved. How do we expand allyship in academia?

- (To all): What types of allyship are typically excluded or not mentioned that you would like to see more awareness about?

- (To faculty members): Faculty members set the tone for the type of environment their courses and research labs will have. What are things that faculty members can do to create inclusive, supportive environments in the classroom, office hours, recitation sessions, and labs? What have you received feedback on that was successful? What are areas for improvement?

- (To faculty members): Allyship necessarily involves advocating for individuals when they are not present, e.g. in faculty meetings and in interactions with other faculty and students. What can faculty do to change departmental culture to be more supportive of underrepresented students in CS?

- (To students): Whereas faculty may have a vision for the type of classroom, lab, or department environment that they want to create, it is the students who inhabit those environments who must enact that vision. What opportunities do students have to exhibit or increase allyship?

- (To all): An important aspect of allyship is to keep improving yourself as an ally. What resources exist for faculty and students to improve as allies?

Following are sample questions that the moderator may pose to the audience in Segment \#3. This will be an opportunity for audience members to share their own insights into allyship so that current and future allies can benefit from their experiences, improve as allies, and pass this knowledge on to others who are not in attendance.

- As a student, in what ways have you benefited from having an academic ally?

- As a student, how have you been failed by academic allies? Did the ally understand their failure? How was it addressed?

- Many members of underrepresented groups enter industry without having had an academic ally while they were a student. In what ways could an academic ally have helped your transition into industry?

- As a faculty member, what are the challenges that you face when it comes to growing the number of allies in your department, and how can you overcome them?

\section{EXPECTATIONS}

We expect a large audience of faculty who consider themselves allies, and those looking to improve as allies. We also expect students to attend so that they can learn more about allyship in academia and provide their perspectives on what they'd like to see. We intend that faculty attendees leave the session understanding that:

- Although allyship can be challenging and risky, it is critical for the future of computing.

- The only way to be an effective ally is to continually learn about allyship and listen to other perspectives.

- Successful allyship must permeate all areas of academic life: not only the classroom and mentoring, but also research labs, departmental events, and the culture as a whole.

We also hope that student attendees leave with a sense that allyship in academia -- among faculty and students alike -- does exist and there is a growing community of those who are making efforts to improve equity in the field of computing.

\section{SUITABILITY FOR A SPECIAL SESSION}

We intend for this special session to be a collaborative conversation across multiple populations and viewpoints - not a dissemination of "authorities" on the subject. Audience participation will be crucial to the success of this session, and we will actively seek the contributions of everyone in attendance so that we all may benefit from hearing each other's insights. The special session format will better allow for this type of discussion and will ensure that many perspectives are heard, not just those of the organizers. Last, the special session format allows us to overcome the limit of having just 3-4 panelists, which allows us to have a group of organizers who could offer a more diverse range of perspectives on allyship. 